\title{
Pressão de aplicação com pulverizador de barra e eficiência de bioinseticidas fúngicos comerciais
}

\author{
Cesar de Oliveira Guimarães ${ }^{(1)}$, Antônia do Carmo Barcelos Correia ${ }^{(1)}$ \\ e Marcelo da Costa Ferreira(1)
}

(1)Universidade Estadual Paulista Júlio de Mesquita Filho, Fac. de Ciências Agrárias e Veterinárias, Dep. de Fitossanidade, Via de Acesso
Paulo Donato Castellane, Km 5, CEP 14884-900 Jaboticabal, SP. E-mail: cesarog@ig.com.br

Resumo - Bioinseticidas fúngicos são aplicados com pulverizadores convencionais e algumas características destes equipamentos podem não ser adequadas e afetar a eficiência dos bioinseticidas. O objetivo deste trabalho foi verificar se pressões usuais, em aplicações com o pulverizador de barra, podem afetar a ação inseticida de conídios de Beauveria bassiana e Metarhizium anisopliae, componentes de produtos comerciais brasileiros. Para isso, suspensões aquosas de três bioinseticidas foram submetidas à passagem pelo equipamento em três pressões $\left(20,40\right.$ e $\left.60 \mathrm{lbf} \mathrm{pol}^{-2}\right)$ e avaliadas quanto ao rompimento, à viabilidade e à virulência dos conídios. Avaliou-se a viabilidade em lâminas de microscopia cobertas com meio de cultura, após incubação a $26 \pm 0,5^{\circ} \mathrm{C}$ e fotoperíodo de 12 horas. A concentração foi determinada por meio de contagens em câmara de Neubauer e a virulência foi avaliada para lagartas de Diatraea saccharalis. Não foram encontradas, nos três produtos, influências significativas em nenhum dos parâmetros. Nas pressões avaliadas, a aplicação com o pulverizador de barra não reduz a viabilidade e nem a virulência dos conídios dos bioinseticidas testados, tampouco provoca destruição dos conídios.

Termos para indexação: Metarhizium anisopliae, Beauveria bassiana, controle microbiano, técnica de aplicação, fungos entomopatogênicos.

\section{Application pressure in boom sprayer and efficiency of commercial fungal bioinsecticides}

\begin{abstract}
Fungal biopesticides are applied with conventional sprayers and this equipment may be inappropriate, and therefore affect field control efficacy of biopesticides. The objective of this research was to find out if the usual pressures used in the boom sprayers can affect the conidia pesticide activity of the Brazilian commercial entomopathogenic fungi Beauveria bassiana and Metarhizium anisopliae. Water fungi suspensions were passed through the equipment under three pressures (20,40 and $60 \mathrm{psi}$ ) and evaluated for viability, virulence and conidia disruption. The viability was evaluated in slides covered by culture media after incubation at $26 \pm 0.5^{\circ} \mathrm{C}$ and 12 hours photophase. The concentration was determined through Neubauer chamber count and the virulence to Diatraea saccharalis caterpillars was evaluated. In the three tested biopesticides no significant effect was found in any parameters. For the tested pressures, the boom sprayer does not affect the viability or virulence and does not destroy the conidia of the evaluated commercial biopesticides.
\end{abstract}

Index terms: Metarhizium anisopliae, Beauveria bassiana, microbial control, application technique, entomopathogenic fungi.

\section{Introdução}

No Brasil, o uso de fungos entomopatogênicos para controle de pragas agrícolas vem se intensificando nos últimos anos, uma vez que pode substituir com vantagens o controle químico em muitas situações. Metarhizium anisopliae (Metshc.) Sorok., fungo com ótimos resultados no controle microbiano, ocorre naturalmente em cerca de 300 espécies de insetos, até mesmo em pragas bastante comuns e prejudiciais, como
Diatraea saccharalis (Fabric.), a broca-da-cana (Alves, 1998).

No Estado de São Paulo, onde a área plantada com cana-de-açúcar é de aproximadamente três milhões de hectares (Fundação Sistema Estadual de Análise de Dados, 2003), o aumento da área colhida, sem queima, tem propiciado o crescimento da população de Mahanarva fimbriolata (Stål.), a cigarrinha-da-raiz (Dinardo-Miranda et al., 2001), contra a qual M. anisopliae vem sendo intensamente utilizado. Além 
desta espécie, Beauveria bassiana (Balsamo) Vuill., um dos fungos mais estudados no controle microbiano de insetos, é agente ativo de diversos produtos comerciais, devido à facilidade de produção massal, estabilidade durante a secagem, compatibilidade com adjuvantes e atoxicidade para vertebrados (Wraight et al., 1998).

A aplicação de patógenos é geralmente feita com os mesmos equipamentos utilizados para agrotóxicos (Steinke \& Giles, 1995; Nilsson \& Gripwall, 1999), porém, estes equipamentos podem não ser adequados para aplicar microrganismos (Steinke \& Giles, 1995). Como observado por Nilsson \& Gripwall (1999), alguns procedimentos e equipamentos utilizados na aplicação de bioinseticidas podem diminuir a viabilidade de agentes microbianos de controle. Além disso, Boucias \& Pendland (1991) verificaram que conídios de M. anisopliae e B. bassiana são recobertos por uma camada de feixes de bastonetes, muito provavelmente envolvida com o processo de penetração do fungo no inseto, a qual foi parcialmente retirada em B. bassiana e Paecilomyces fumosoroseus, quando suspensões de conídios destes fungos foram homogeneizadas em agitador tipo Vórtex.

Um dos equipamentos mais utilizados na aplicação destes bioinseticidas é o pulverizador de barra, composto basicamente por: um chassi, o qual suporta o reservatório de calda (ou tanque); pelo filtro de linha, localizado antes da bomba, responsável por evitar que partículas sólidas possam danificá-la ou causar entupimento nos bicos; pela bomba, normalmente de pistões, que gera um fluxo unidirecional e com alta capacidade de pressurização do sistema; pelo regulador de pressão que permite que o excesso de volume bombeado retorne ao tanque; pelo manômetro, que indica a pressão da calda no sistema; e pelo registro de abertura e fechamento de fluxo da calda, que permite o retorno da suspensão ao tanque (Matuo, 1990).

Apesar do risco de componentes dos sistemas de pulverização causarem danos a microrganismos, existem poucas pesquisas sobre a tecnologia de aplicação de entomopatógenos (Alves et al., 1998). Mesmo havendo dúvidas a respeito da inocuidade para os microrganismos, estes equipamentos vêm sendo cada vez mais empregados para aplicação de inseticidas fúngicos produzidos por biofábricas no Estado de São Paulo.

O objetivo deste trabalho foi verificar se as pressões utilizadas durante a aplicação com um pulverizador de barra afetam a viabilidade, virulência ou rompem os conídios, reduzindo a concentração do bioinseticida aplicado, parâmetros importantes para a eficiência de bioinseticidas fúngicos comerciais.

\section{Material e Métodos}

A limpeza e calibração dos pulverizadores foram realizadas no Galpão da Fazenda de Ensino e Pesquisa. O preparo das suspensões e a coleta das amostras foram realizadas em área do Dep. de Engenharia Rural. As análises foram conduzidas no Laboratório de Entomopatógenos do Dep. de Fitossanidade, todos pertencentes ao campus da Unesp de Jaboticabal. Foram utilizados pulverizadores de barra modelo KO $600 \mathrm{IH} \mathrm{12,}$ com capacidade para $600 \mathrm{~L}$, equipados com bomba de três pistões e bicos de polímero marca Magno 11002, cedidos pela empresa KO Implementos Agrícolas Ltda., de Jaboticabal, SP.

Nos três ensaios, o equipamento ainda não havia sido utilizado e estava livre de contaminação por agrotóxicos ou outros entomopatógenos. Foram utilizados três produtos comerciais, sendo um à base de Beauveria bassiana, o Boveril (isolado B103), e dois à base de Metarhizium anisopliae, o Metarril (isolado M103) e o Metarriz Biocontrol (isolado B23). As amostras recebidas foram armazenadas em refrigerador (aproximadamente $8^{\circ} \mathrm{C}$ ) até o momento da instalação dos ensaios.

Para cada produto realizou-se um ensaio com delineamento inteiramente casualizado com seis repetições e quatro tratamentos: controle, cuja pressão é zero, pressão de 20 libras força por polegada quadrada ( $\left(\mathrm{bf} \mathrm{pol}^{-2}\right)$, pressão de $40 \mathrm{lbf}$ pol$^{-2}$ e pressão de $60 \mathrm{lbf} \mathrm{pol}^{-2}$. As pressões foram escolhidas com base na faixa de trabalho do equipamento e dos bicos mais comumente utilizados pelos agricultores, no campo. Os dados foram submetidos à análise de variância e as médias comparadas pelo teste de Tukey. Os dados de mortalidade e viabilidade foram transformados em arco seno $\sqrt{x / 100}$.

As viabilidades dos produtos no momento do recebimento das amostras foram de 93,2\%, 94,6\% e 95,1\%, respectivamente, para Boveril, Metarril e Metarriz Biocontrol. No momento da instalação dos bioensaios, as viabilidades foram, respectivamente, de $89,1 \%, 85,9 \%$ e 94,0\%. Essas diferenças são justificadas, provavelmente, pelos diferentes tempos de armazenamento em refrigerador, de no máximo quatro semanas.

As suspensões de conídios foram preparadas de acordo com a recomendação dos fabricantes, em um volume de $200 \mathrm{~L}$ de água por ensaio utilizando-se $2 \mathrm{~kg}$ de 
produto comercial, com adição de $0,25 \%$ de espalhante adesionante (Extravon). Foi usada água potável da rede do campus de Jaboticabal, proveniente de poço artesiano profundo. As condições de cada bioensaio estão na Tabela 1 .

Dentro do tanque do pulverizador, a suspensão foi agitada por 15 minutos e, em seguida, coletaram-se amostras do tratamento testemunha, diretamente no tanque. Para coletar as amostras da suspensão pressurizada, as pressões foram ajustadas e o líquido foi pulverizado sobre um anteparo de vidro, posicionado a $50 \mathrm{~cm}$ do bico de pulverização, e acondicionado em potes de plástico de $250 \mathrm{~mL}$. Entre coletas das amostras de diferentes pressões, houve um intervalo de dois minutos com a vazão de calda aberta, para descartar a suspensão remanescente nas tubulações e que havia sido submetida apenas à pressão anterior.

Foi avaliado se, durante a passagem pelo equipamento, ocorreu rompimento extenso da parede de conídios a ponto de causar o extravasamento de todo seu conteúdo citoplasmático, estimando-se sua concentração nas suspensões de cada tratamento. Para tanto, das amostras acondicionadas nos potes de plástico, eram retiradas, após agitação manual, alíquotas de $5 \mathrm{~mL}$, as quais eram colocadas em tubos de ensaio com duas gotas de corante ácido (solução estoque: $1 \mathrm{~g}$ de Metil Blue em $10 \mathrm{~mL}$ de ácido lático; desta, foi tomado $1 \mathrm{~mL}$ e adicionado a $29 \mathrm{~mL}$ de ácido lático) para prevenir o crescimento de outros microrganismos. Destas sub-amostras, os conídios em câmara de Neubauer foram contados em 20 campos de $0,04 \mathrm{~mm}^{2}$, preparando-se 2 lâminas de cada amostra.

Para verificar se a pressão pode alterar a viabilidade dos conídios, foram realizados testes com as suspensões, antes e depois de serem submetidas às três pressões. Foram utilizadas lâminas de microscopia cobertas com $4 \mathrm{~mL}$ de meio ágar-água para $B$. bassiana, e batata-dextrose-ágar para M. anisopliae, adicionados com pentabiótico cloranfenicol na concentração de $0,125 \mathrm{mg} \mathrm{mL}^{-1}$. Sobre cada lâmina adicionou-se uma gota de suspensão, em três áreas distintas.

As lâminas foram acondicionadas em câmaras úmidas, permanecendo em estufa a $26 \pm 0,5^{\circ} \mathrm{C}$ por 12 horas, com Boveril e Metarriz Biocontrol, e por 18 horas, com Metarril (tempos indicados por testes preliminares). Uma gota do corante ácido, utilizado também para paralisar a germinação, foi adicionada às lâminas ao final do tempo pré-determinado. O conídio foi considerado viável quando seu tubo germinativo atingiu pelo menos o comprimento (M. anisopliae) ou o diâmetro (B. bassiana) do conídio. A viabilidade foi avaliada contando-se, ao microscópio, 300 conídios por lâmina, totalizando 900 conídios por repetição, com aumento de 400X.

Para verificar se a pressão pode afetar a agressividade do fungo, foram utilizadas, para cada uma das seis repetições, dez lagartas de Diatraea saccharalis de 3 - ou 4은 ínstar, fornecidas pela Usina Santa Adélia, Jaboticabal, SP. Elas foram criadas em dieta contendo açúcar $(27 \mathrm{~g})$, farelo de soja (40 g), gérmen de trigo (13 g), solução vitamínica (3 mL), carragenato ( $10 \mathrm{~g})$, ácido ascórbico $(0,4 \mathrm{~g})$, ácido acético (3,5 $\mathrm{mL})$, formol $(1 \mathrm{~mL})$, fungicida nipagin $(2,4 \mathrm{~g}) \mathrm{e}$ água $(420 \mathrm{~mL})$. Ainoculação foi feita por meio de imersão das lagartas por 5 segundos nas amostras das suspensões, utilizando-se uma peneira. Após cada imersão, a base da peneira era apoiada em papel toalha para retirar o excesso de líquido. A seguir, as lagartas eram transferidas, com auxílio de pincel, para caixas de plástico com $6 \mathrm{~cm}$ de diâmetro por $1,5 \mathrm{~cm}$ de altura, forradas com papel-filtro umedecido com água destilada, e mantidas em estufa tipo BOD a $26 \pm 05^{\circ} \mathrm{C}$ e fotófase de 14 horas. Após 48 horas, o papel-filtro foi retirado. Devido à presença do fungicida em sua composição, a dieta só foi oferecida às lagartas dois dias após a inoculação (Andrioli, 2003). Diariamente, as lagartas mortas eram

Tabela 1. Condições verificadas durante o preparo das suspensões e coleta das amostras.

\begin{tabular}{|c|c|c|c|}
\hline Parâmetro & Boveril & Metarril & Metarriz Biocontrol \\
\hline Temperatura ambiente máxima $\left({ }^{\circ} \mathrm{C}\right)$ & 29,5 & 29,5 & 30,0 \\
\hline Temperatura ambiente mínima $\left({ }^{\circ} \mathrm{C}\right)$ & 28,5 & 29,1 & 28,5 \\
\hline Umidade relativa do ar máxima (\%) & 31,0 & 31,0 & 40,0 \\
\hline Umidade relativa do ar mínima (\%) & 25,0 & 25,0 & 35,0 \\
\hline Duração do preparo da suspensão (min) & 50,0 & 50,0 & 15,0 \\
\hline Duração da coleta das amostras (min) & 25,0 & 25,0 & 30,0 \\
\hline Temperatura da água antes da colocação do fungo $\left({ }^{\circ} \mathrm{C}\right)$ & 28,5 & 28,5 & 29,0 \\
\hline Temperatura da suspensão após coleta da última amostra $\left({ }^{\circ} \mathrm{C}\right)$ & 30,0 & 30,0 & 31,0 \\
\hline Rotação do motor (rpm) & $1.300,0$ & $1.300,0$ & $1.300,0$ \\
\hline
\end{tabular}


retiradas e mergulhadas em álcool etílico $70 \%$ por 30 segundos, para desinfecção externa do tegumento, enxaguadas duas vezes em água estéril e, então, colocadas em câmaras úmidas para emergência e esporulação do fungo, confirmando-se assim a causa da morte.

\section{Resultados e Discussão}

Pelos resultados obtidos para a viabilidade dos conídios dos três produtos comerciais, verificou-se que as pressões utilizadas não reduziram a capacidade de germinação dos fungos (Tabela 2). Este relato está de acordo com Garcia (2003) que, trabalhando com a pressurização de $M$. anisopliae, constatou que pressões de até $200 \mathrm{lbf} \mathrm{pol}^{-2}$ não diminuíram sua capacidade de germinação, e, também, com o observado por Nilsson \& Gripwall (1999), pois somente pressões acima de $420 \mathrm{lbf} \mathrm{pol}^{-2}$ influenciaram negativamente a viabilidade de Verticillium lecanii. Estes últimos observaram ainda uma elevação de temperatura de $12^{\circ} \mathrm{C}$ na suspensão, durante um tempo de agitação de 30 minutos, no qual a viabilidade do fungo foi reduzida de aproximadamente $100 \%$ para $50 \%$, comparado a um tempo de 2 minutos, em que não houve redução. Entretanto, neste trabalho, a maior elevação de temperatura da suspensão foi de $2^{\circ} \mathrm{C}$. Em ambos os casos, a geração de calor é conseqüência do atrito das partes móveis da bomba, do líqui- do no interior das tubulações e da pressurização do sistema hidráulico.

Apesar de o tempo de agitação não ter sido objeto de análise estatística, pode-se inferir que também não afetou a viabilidade do fungo, pois as amostras da pressão

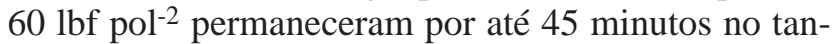
que, enquanto as amostras da testemunha sofreram uma agitação de apenas 15 minutos. Uma causa dessa diferença pode ser o volume de suspensão relativamente alto, utilizado no ensaio. Nos experimentos de Nilsson \& Gripwall (1999), o volume inicial foi de 10\% (30 L) do volume total do tanque, enquanto que neste trabalho, o volume de suspensão inicial foi de $33 \%$ (200 L). Outra causa pode ter sido a pressão de trabalho do pulverizador testado. Segundo Nilsson \& Gripwall (1999), a pressão chegou a $700 \mathrm{lbf} \mathrm{pol}^{-2}$ e neste trabalho, a $60 \mathrm{lbf} \mathrm{pol}^{-2}$. Ao serem comparadas as viabilidades dos três produtos, constata-se que Metarriz apresentou valores maiores, fato possivelmente ocorrido devido ao menor tempo de armazenamento no refrigerador e à necessidade de padronização no tempo de incubação dos conídios durante o teste de viabilidade.

Assim como a viabilidade, a concentração e a virulência também não foram afetadas pelas diferentes pressões, no tocante aos três bioinseticidas (Tabela 2).

A necessidade da colocação de filtros especiais, como sugerido por Alves et al. (1998) e Garcia (2003), para evitar entupimentos e garantir o perfeito funcionamento

Tabela 2. Concentração de conídios, porcentagem de viabilidade e mortalidade (médiasterro-padrão), obtidas de suspensão aquosa preparada com os bioinseticidas comerciais Boveril, Metarril e Metarriz Biocontrol antes e após aplicação com pulverizador de barra em três pressões.

\begin{tabular}{|c|c|c|c|c|}
\hline Bioinseticida & $\begin{array}{c}\text { Tratamento } \\
\text { (pressões em lbf } \text { pol}^{-2} \text { ) }\end{array}$ & $\begin{array}{c}\text { Concentração } \\
\left(\text { conídios } \times 10^{5} \mathrm{~mL}^{-1}\right)\end{array}$ & $\begin{array}{l}\text { Viabilidade }^{(2)} \\
(\%)\end{array}$ & Mortalidade $^{(2)}$ \\
\hline Boveril & $\begin{array}{r}0 \\
20 \\
40 \\
60\end{array}$ & $\begin{array}{l}3,09 \pm 1,29 \\
3,17 \pm 0,97^{\mathrm{ns}} \\
3,15 \pm 1,06^{\mathrm{ns}} \\
3,26 \pm 1,11^{\mathrm{ns}}\end{array}$ & $\begin{array}{l}69,85 \pm 0,52 \\
68,55 \pm 1,57^{\mathrm{ns}} \\
68,76 \pm 0,79^{\mathrm{ns}} \\
67,69 \pm 0,57^{\mathrm{ns}}\end{array}$ & $\begin{array}{l}31,75 \pm 2,84 \\
43,99 \pm 6,88^{\mathrm{ns}} \\
36,64 \pm 6,44^{\mathrm{ns}} \\
42,07 \pm 3,65^{\mathrm{ns}}\end{array}$ \\
\hline $\mathrm{CV}(\%)$ & & 8,58 & 3,43 & 27,06 \\
\hline Metarril & $\begin{array}{r}0 \\
20 \\
40 \\
60\end{array}$ & $\begin{array}{l}4,69 \pm 0,23 \\
4,40 \pm 0,07^{\mathrm{ns}} \\
4,56 \pm 0,14^{\mathrm{ns}} \\
4,53 \pm 0,11^{\mathrm{ns}}\end{array}$ & $\begin{array}{l}65,33 \pm 0,99 \\
67,18 \pm 0,84^{\text {ns }} \\
66,43 \pm 1,59^{\text {ns }} \\
63,61 \pm 1,11^{\text {ns }}\end{array}$ & $\begin{array}{l}37,03 \pm 4,28 \\
50,03 \pm 3,88^{\text {ns }} \\
42,78 \pm 7,38^{\text {ns }} \\
31,71 \pm 5,94^{\text {ns }}\end{array}$ \\
\hline $\mathrm{CV}(\%)$ & & 8,00 & 4,35 & 33,67 \\
\hline Metarriz Biocontrol & $\begin{array}{r}0 \\
20 \\
40 \\
60\end{array}$ & $\begin{array}{l}1,09 \pm 0,07 \\
0,96 \pm 1,63^{\mathrm{ns}} \\
0,99 \pm 1,62^{\mathrm{ns}} \\
1,32 \pm 0,14^{\mathrm{ns}}\end{array}$ & $\begin{array}{l}75,71 \pm 0,76 \\
74,90 \pm 1,04^{\text {ns }} \\
72,19 \pm 1,35^{\text {ns }} \\
73,37 \pm 0,83^{\text {ns }}\end{array}$ & $\begin{array}{l}46,71 \pm 7,41 \\
36,58 \pm 5,35^{\text {ns }} \\
44,64 \pm 6,37^{\text {ns }} \\
43,07 \pm 7,76^{\text {ns }}\end{array}$ \\
\hline $\mathrm{CV}(\%)$ & & 21,05 & 3,38 & 38,57 \\
\hline
\end{tabular}

${ }^{(1)}$ Dados não transformados. ${ }^{(2)}$ Valores transformados em arco seno $\sqrt{x / 100} \cdot{ }^{\text {ns }}$ Não-significativo. 
do sistema, não ocorreu neste experimento. A aplicação transcorreu normalmente durante todos os ensaios, com todos os componentes originais do pulverizador. Observou-se acúmulo de material particulado nos filtros do sistema, mas isso não prejudicou a fluidez da calda e a pulverização. Além disso, a concentração inalterada da suspensão desde a primeira até a última coleta, em todos os ensaios, mostrou que os conídios não estavam sendo retidos de maneira significativa nos filtros presentes no equipamento.

Os valores obtidos para a virulência foram relativamente baixos. Isso foi provavelmente causado pela baixa concentração das suspensões preparadas para os ensaios. Andrioli (2003), trabalhando com B. bassiana, obteve mortalidades de até $100 \%$ para lagartas de D. saccharalis, utilizando suspensões com concentração da ordem de $10^{7}$ conídios $\mathrm{mL}^{-1}$. Neste trabalho, a concentração dos três produtos testados foi de $10^{5}$ conídios $\mathrm{mL}^{-1}$. Também pode ter contribuído para a baixa mortalidade, a inadequação dos isolados dos bioinseticidas para a broca-da-cana, fato ilustrado por Arcas et al. (1999). Os autores compararam a ação de dois isolados de $B$. bassiana $\left(\mathrm{Bb}_{5}\right.$ e $\left.\mathrm{Bb}_{1}\right)$ para $D$. saccharalis e obtiveram, respectivamente, mortalidades de $82,5 \%$ e $21,3 \%$.

A escolha do inseto para os testes foi baseada na sua disponibilidade em quantidades suficientes para realização dos ensaios e na suscetibilidade ao patógeno. Apesar de os fungos dos bioinseticidas testados serem patogênicos para $D$. saccharalis, os fabricantes não os recomendam para controle desta praga.

\section{Conclusão}

A aplicação nas pressões de 20 a 60 lbf poll- ${ }^{-2}$ com pulverizador de barra, não afeta a virulência e a viabilidade, nem rompe os conídios dos bioinseticidas Boveril, Metarril e Metarriz Biocontrol.

\section{Agradecimentos}

À KO Implementos Agrícolas Ltda., de Jaboticabal, SP, pelo empréstimo dos pulverizadores; à Itaforte Industrial de Bioprodutos Agro-Florestais Ltda., de Itapetininga, SP, e à Biocontrol Sistema de Controle Biológico Ltda., de Sertãozinho, SP, pela doação das amostras dos bioinseticidas utilizados; à Usina Santa Adélia, de Jaboticabal, SP, pelo fornecimento das lagartas de D. saccharalis; ao Dep. de Engenharia Rural da
FCAV/Unesp, por ceder a área onde foram realizadas as amostragens; aos funcionários da Fazenda de Ensino e Pesquisa da FCAV/Unesp-Jaboticabal, pelo auxílio no manejo dos pulverizadores; aos colegas Alexandre Hiromiti Sano, Edimara Aparecida Francisco, Eliane Ribeiro Cardoso, Gustavo Bissoli e à técnica de laboratório Sandra Sesso, pelo auxílio no laboratório; aos Profs. Dr. Antônio Carlos Monteiro e Dr. Ely Nahas, pelas sugestões ao manuscrito do trabalho; ao Prof. Antônio Carlos Barbosa, pelo auxílio com as análises estatísticas.

\section{Referências}

ALVES, S.B. Fungos entomopatogênicos. In: ALVES, S.B. (Ed.). Controle microbiano de insetos. Piracicaba: Fealq, 1998. p.289381.

ALVES, S.B.; PEREIRA, R.M.; ALMEIDA, J.E.M.; LARANJEIRO, A.J. Equipamentos para aplicação de inseticidas microbianos. In: ALVES, S.B. (Ed.). Controle microbiano de insetos. Piracicaba: Fealq, 1998. p.269-287.

ANDRIOLI, J.L. Alterações na viabilidade e virulência de Beauveria bassiana (Bals) Vuill. causadas por técnicas usadas para obtenção de conídios e formulação de um bioinseticida. 2003. 68p. Tese (Doutorado) - Universidade Estadual Paulista, Araraquara.

ARCAS, J.A.; DIAZ, B.M.; LECUONA, R.E. Bioinsecticidal activity of conidia and dry mycelium preparations of two isolates of Beauveria bassiana against the sugarcane borer Diatraea saccharalis. Journal of Biotechnology, v.67, p.151-158, 1999.

BOUCIAS, D.G.; PENDLAND, J.C. Attachment of mycopathogens to cuticule: the initial event of mycosis in anthropod host. In: COLE, G.T.; HOCH, H.C. (Ed.). The fungal spore disease initiation in plants and animals. New York: Plenum Press, 1991. p.101-125.

DINARDO-MIRANDA, L.L.; MOSSIM, G.C.; DURIGAN, A.M.P.R.; BARBOSA, V. Controle químico de cigarrinha das raízes, Mahanarva fimbriolata, em cana-de-açúcar. STAB, v.19, p.20-23, 2001.

FARIA, M.R.; MAGALHÃES, B.P. O uso de fungos entomopatogênicos no Brasil. Biotecnologia Ciência e Desenvolvimento, v.22, p.18-21, 2001.

Fundação Sistema Estadual de Análise de Dados. Sensor Rural. São Paulo. Disponível em: <www.seade.gov.br/cgi-bin/dirasv98/ sensor_01.ksh?> Acesso em: 15 jan. 2004.

GARCIA, L.C. Interferência da pressurização da calda de pulverização sobre microorganismos entomopatogênicos. 2003. 52p. Dissertação (Mestrado) - Universidade Estadual Paulista, Botucatu. 
MATUO, T. Técnicas de aplicação de defensivos agrícolas. Jaboticabal: Funep, 1990. 139p.

NILSSON, U.; GRIPWALL, E. Influence of application technique on the viability of the biological control agents Verticillium lecanii and Steinernema feltiae. Crop Protection, v.18, p.53-59, 1999.

STEINKE, E.; GILES, D.K. Delivery Systems for Biorational Agents. In: HALL, F.R.; BARRY, J.W. (Ed.). Biorational pest control agents: formulations and delivery. Washington, DC: American Chemical Society, 1995. p.80-94.

WRAIGHT, S.P.; CARRUTHERS, R.I.; BRADLEY, C.A.; JARONSKI, S.T.; LACEY, L.A.; WOOD, P. Pathogenicity of entomopathogenic fungi Paecilomyces spp. and Beauveria bassiana against the silverleaf whitefly, Bemisia argentifolii. Journal of Invertebrate Pathology, v.71, p.217-226, 1998.

$\overline{\text { Recebido em } 11 \text { de fevereiro de } 2004 \text { e aprovado em } 8 \text { de setembro de } 2004}$

Pesq. agropec. bras., Brasília, v.39, n.12, p.1177-1182, dez. 2004 